\title{
Automatic recording of individual oestrus vocalisation in group-housed dairy cattle: development of a cattle call monitor
}

\author{
V. Röttgen ${ }^{1,2 a}$, P. C. Schön ${ }^{1 a}$, F. Becker², A. Tuchscherer ${ }^{3}$, C. Wrenzycki ${ }^{4}$, S. Düpjan ${ }^{1+}$ and \\ B. Puppe ${ }^{1,5}$
}

${ }^{1}$ Institute of Behavioural Physiology, Leibniz Institute for Farm Animal Biology (FBN), Wilhelm-Stahl-Allee 2, D-18196 Dummerstorf, Germany; ${ }^{2}$ Institute of Reproductive Biology, Leibniz Institute for Farm Animal Biology (FBN), Wilhelm-Stahl-Allee 2, D-18196 Dummerstorf, Germany; ${ }^{3}$ nstitute of Genetics and Biometry, Leibniz Institute for Farm Animal Biology (FBN), Wilhelm-Stahl-Allee 2, D-18196 Dummerstorf, Germany; ${ }^{4}$ Clinic for Veterinary Obstetrics, Gynecology and Andrology, Faculty of Veterinary Medicine, Justus-Liebig-University Giessen, Frankfurter Straße 106, D-35392 Giessen, Germany; ${ }^{5}$ Behavioural Sciences, Faculty of Agricultural and Environmental Sciences, University of Rostock, Justus-von-Liebig-Weg 7, D-18059 Rostock, Germany

(Received 23 November 2018; Accepted 20 June 2019; First published online 1 August 2019)

Oestrus detection remains a problem in the dairy cattle industry. Therefore, automatic detection systems have been developed to detect specific behavioural changes at oestrus. Vocal behaviour has not been considered in such automatic oestrus detection systems in cattle, though the vocalisation rate is known to increase during oestrus. The main challenge in using vocalisation to detect oestrus is correctly identifying the calling individual when animals are moving freely in large groups, as oestrus needs to be detected at an individual level. Therefore, we aimed to automate vocalisation recording and caller identification in grouphoused dairy cows. This paper first presents the details of such a system and then presents the results of a pilot study validating its functionality, in which the automatic detection of calls from individual heifers was compared to video-based assessment of these calls by a trained human observer, a technique that has, until now, been considered the 'gold standard'. We developed a collar-based cattle call monitor (CCM) with structure-borne and airborne sound microphones and a recording unit and developed a postprocessing algorithm to identify the caller by matching the information from both microphones. Five group-housed heifers, each in the perioestrus or oestrus period, were equipped with a CCM prototype for 5 days. The recorded audio data were subsequently analysed and compared with audiovisual recordings. Overall, 1404 vocalisations from the focus heifers and 721 vocalisations from group mates were obtained. Vocalisations during collar changes or malfunctions of the CCM were omitted from the evaluation. The results showed that the CCM had a sensitivity of $87 \%$ and a specificity of $94 \%$. The negative and positive predictive values were $80 \%$ and $96 \%$, respectively. These results show that the detection of individual vocalisations and the correct identification of callers are possible, even in freely moving group-housed cattle. The results are promising for the future use of vocalisation in automatic oestrus detection systems.

Keywords: Bos taurus, oestrus detection, vocalisation, caller identification, bioacoustics

\section{Implications}

Our aim is to establish vocalisation as an innovative parameter for oestrus detection in dairy cows. We present a combined hardware and software solution for individual vocalisation recording in group-housed cattle for use as an additional oestrus indicator on dairy farms. This could be of interest for researchers, farmers and the farm equipment industry. We show that the automatic detection of individual vocalisations and the correct identification of callers are possible, even in freely moving group-housed cows. These results

These authors contributed equally to this work.

† E-mail: duepjan@fbn-dummerstorf.de are promising for automatic oestrus detection systems and, beyond that, for further applications in terms of monitoring animal health and welfare status.

\section{Introduction}

Oestrus detection is an essential component of successful reproduction management for dairy cattle. In the last several decades, automatic devices have been developed to assist farmers in this task. The most frequently used parameter acquired by automatic oestrus detection devices is an increase in physical activity, as measured by pedometers 
attached to the leg of the animal or by other devices located at its neck (Rutten et al., 2013). Another behaviour that is currently monitored in the context of oestrus detection is rumination (Reith et al., 2012), with duration of rumination and changes in the time spent ruminating in certain daytime intervals being the main parameters. Additional commercially available devices detect mounting behaviour as part of oestrus behaviour (Walker et al., 1996). These mounting detectors are attached to the cow's back and detect the mounting activity of herd members to identify cows that show standing heat behaviour, that is, those who will tolerate being mounted by a potential mating partner. However, although these different parameters have been implemented in commercially available automatic oestrus detection devices, farmers are still facing significant economic losses due to undetected oestrus cycles. Beyond oestrus detection, physical activity and rumination are also typically used for health monitoring in commercially available devices.

Another behavioural parameter of farm animals that is monitored (albeit rarely) is vocal behaviour. Vocalisations convey not only semantic information (e.g., 'I am here' in contact calls) but also contextual information about, for example, body size, sex, identity, state of the sexual cycle, rank and exhaustion (Fischer et al., 2004; Mielke and Zuberbühler, 2013; Pitcher et al., 2014). Pigs produce context-specific distress calls (screams) when confronted with acute, severe stressors (Puppe et al., 2005; Düpjan et al., 2008), which can be detected automatically to monitor their welfare (Schön et al., 2001; Schön et al., 2004), but they also demonstrate subtle variations in vocalisation indicative of their affective state (Leliveld et al., 2016) and emotional reactivity (Leliveld et al., 2017). For cattle, it is known that vocalisation rates increase at the day of oestrus (Schön et al., 2007; Dreschel et al., 2014) and that the vocalisation climax occurs shortly before or at the climax of oestrus behaviour (Röttgen et al., 2018). Considering that endogenous hormone secretion is likewise associated with oestrus behaviour (Lyimo et al., 2000; Aungier et al., 2015), vocalisation can serve as an indirect indicator of endogenous hormonal changes and therefore as a potential additional parameter for automatic oestrus detection.

There are some examples of successful acoustic monitoring of farm animals, for instance, a commercial device for cough detection in pigs. The device records and analyses incoming sounds via stationary microphones, identifies coughs and triangulates the housing compartment where they originate from (Silva et al., 2008). Meen et al. (2015) proposed a similar system to monitor animal welfare in cattle. However, while the assignment to a specific individual animal (henceforth termed 'caller identification') is not essential for cough detection in pigs, it is essential for oestrus detection. Yajuvendra et al. (2013) tried to solve the problem of caller identification by using individual structural characteristics in vocalisations. Similar techniques have been used in wildlife species (e.g., in blue monkeys: Mielke and Zuberbühler, 2013). However, these techniques require high-quality samples of vocalisations for each individual animal to enable the identification of individual call characteristics. For vocalisation to be used in automatic oestrus detection devices on farms, it must be easy to use, and caller identification must be accurate. Therefore, a system based on individual samples seems unlikely to be a feasible solution. Another possibility that has been investigated is to equip each animal with a microphone (as tested in chipmunks: Couchoux et al., 2015). In group-housed animals, however, caller identification might be inaccurate, as group mates can vocalise close to another individual's microphone (but see Gill et al., 2016, for a technical solution in zebra finches).

Automatic detection of specific behaviours on an individual level is the first step towards implementing such detection in technical devices that support farmers. A very challenging task - beyond vocalisation detection itself - is to develop algorithms that make the gained data easily assessable on farms. Algorithms must compensate for individual differences and must be self-adaptive to individual variation to accurately identify a large percentage of animals without manual adjustments. Therefore, these algorithms must be based on fundamental knowledge of individual variation, the detection errors of the system and the timing of the behaviours in relation to the event (e.g., oestrus) they are developed to detect.

If calls can be detected and the caller correctly identified with sufficient sensitivity and specificity, future developments can implement the data to detect peaks in individual vocalisation rates to assist in oestrus detection. Therefore, we developed a collar-based cattle call monitor (CCM) with structure-borne (SBM) and airborne sound microphones $(\mathrm{ABM})$ and a recording unit that uses a postprocessing algorithm to identify the caller by matching the information from both microphones. The aim of this paper is to first present the developed system and to then present the validation of its functionality in a pilot study in which the automatic detection of calls from individual heifers was compared to a videobased assessment of these calls by a trained human observer, a technique that has, until now, been considered the 'gold standard'.

\section{Materials and methods}

\section{Development of the Cattle Call Monitor}

Collar. The CCM was developed based on a regular cattle neck strap with a plastic locking system (see Figure 1). To ensure proper contact with the animal's neck, an elastic band was inserted close to the lock. A box for technical equipment was mounted on the collar next to the inserted elastic band. This box included an ABM (Elecret-Condenser-Microphone EMY-9765P, EKULIT Elektrotechnik, Ostfildern, Germany; frequency range: 30 to $16000 \mathrm{~Hz}$ ), an SBM for recording structure-borne sounds (MicW i456 Cardioid Recording Microphone, frequency range: $100 \mathrm{~Hz}$ to $10 \mathrm{kHz}$; MicW Audio, Beijing, China), an energy supply, a microSD card (Verbatim 8 GB microSDHC Class 10; Verbatim Ltd., Charlotte, NC, USA) as a recording unit and a processing unit 


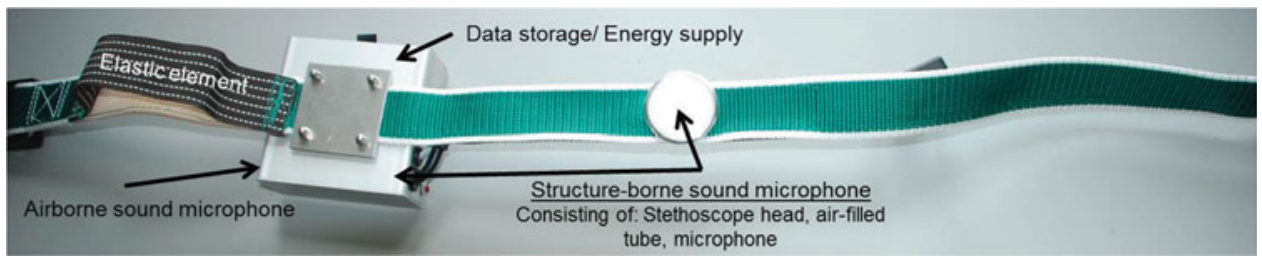

Figure 1 (colour online) Picture of the collar-based cattle call monitor (CCM). The essential components and their location at the collar are marked either with arrows or written on the component itself.

(TMS320C5515 eZdsp; Spectrum Digital, Inc., Stafford, TX, USA). To capture structure-borne sounds, a stethoscope head was mounted on the inside of the strap and connected via a plastic air-filled tube to the SBM in the box. The stethoscope head was positioned on the left side of the animal's neck over the cleido-occipital muscle and the cervical part of the trapezius muscle. This area allows firm contact between the stethoscope head and the animal's body and vibrates noticeably when an animal vocalises. Audio data were sampled using the stereo line-in attached to the processing unit. One channel was used for the SBM, and the other was used for the ABM. Data from both microphones were sampled at a rate of $22048 \mathrm{~Hz}$ to transform the analogue sound signal into digital data. Data were saved on the microSD card in files of a fixed size of $44112 \mathrm{~KB}$. Every day, the collars were detached from the animals, so that the data could be read out and stored in a central storage location.

Machine-aided vocalisation detection. The stored data were analysed on a stationary computer using an algorithm implemented in custom-built software (see Supplementary Figure S1) coded in LabVIEWTM (LabVIEW 2014, Senvice Pack 1, Version 14.0.1f3; National Instruments, Austin, TX, USA). In general, automatic recording of vocalisations requires the separation of sound events from silent periods and the subsequent identification of animal calls amongst these sound events. As the exact start and end of a sound can hardly be detected automatically under on-farm recording conditions, this can only be done by analysing the incoming signal from the microphone in segments called 'windows'. We set the window size at 1024 data points, which at our sampling rate of $22048 \mathrm{~Hz}$ (i.e., 22048 data points per second) corresponds to a duration of $46.4 \mathrm{~ms}$. Windows that exceeded the amplitude threshold of $1 \%$ of the maximum voltage qualified as a sound event and were temporarily saved to the buffer and merged with all adjacent windows passing the criterion. Sound events ended once a window fell below the amplitude threshold. This procedure for the event-oriented recording of animal calls was previously developed by our research group (Schön et al., 2004). In addition to those from the ABM, the SBM signals were also saved in the buffer.

To qualify as a potential call and be permanently stored, the recorded sound events had to meet three criteria (see Figure 2): (1) The signal of the ABM had to be in the frequency range of 50 to $2000 \mathrm{~Hz}$. (2) The duration of the ABM sound event had to be between 0.5 and $10 \mathrm{~s}$. (3) The corresponding signal of the SBM had to have, on average, more than five zero crossings per window. These criteria were implemented to record vocalisations from only the focus animal and to prevent false recordings. Criterion (1) prevented the recording of low-amplitude background noise, for example, noise from heavy farm machinery such as feed mixing vehicles. Criterion (2) excluded normal/high-amplitude short-duration background noise, for example, noise from rattling of the equipment. Criterion (3) ensured that both microphones ( $A B M$ and SBM) were active and therefore should have excluded calls from group mates (see Figure 3 ). If all three criteria (frequency range, duration and zero crossings) were met, the signals from both microphones were stored in a separate file together with a timestamp. Due to the windowing of the data, a single vocalisation could be split into two separate 'calls' (split call) if one window in the middle failed the criteria, but the remaining rest of the vocalisation met the criteria of the algorithm again. These preselected vocalisations were analysed for the remaining ambient sounds by a human observer, and any such sounds were manually deleted $(18 \%$ of the original detections by the $(C M)$.

\section{Validation of the Cattle Call Monitor (pilot study)}

Animals and housing. Recordings were conducted at the Experimental Facility for Cattle at the Leibniz Institute for Farm Animal Biology in Dummerstorf, Germany. We observed five CCM-wearing German Holstein heifers from three groups of four animals each (group 1: three focus animals, i.e., heifers in oestrus or perioestrus; group 2: one focus animal and group 3: one focus animal). The observed animals were randomly selected from a larger group of heifers at the institute. The groups were successively housed in a 5- by 10-m pen with wood shavings as the bedding material. The pen was equipped with a self-locking feeding fence and three freely accessible drinking bowls. The animals were fed once a day with a total mixed ration ad libitum.

All procedures were approved by the federal state of Mecklenburg-Western Pomerania (LALLF M-V/TSD/7221.32.1-021/13).

Video observation. Audiovisual data were recorded using two cameras (EverFocus HDTV, EverFocus Co. Ltd., New Taipei City, Taiwan) positioned on the left and right of the pen and a microphone (Sennheiser MKE600; Sennheiser Electronic GmbH \& Co. KG, Wedemark, Germany) positioned under the ceiling above the heifers, all of which were attached to a digital video recorder (EDR HD-4H4, EverFocus Co. Ltd., New Taipei City, Taiwan). 


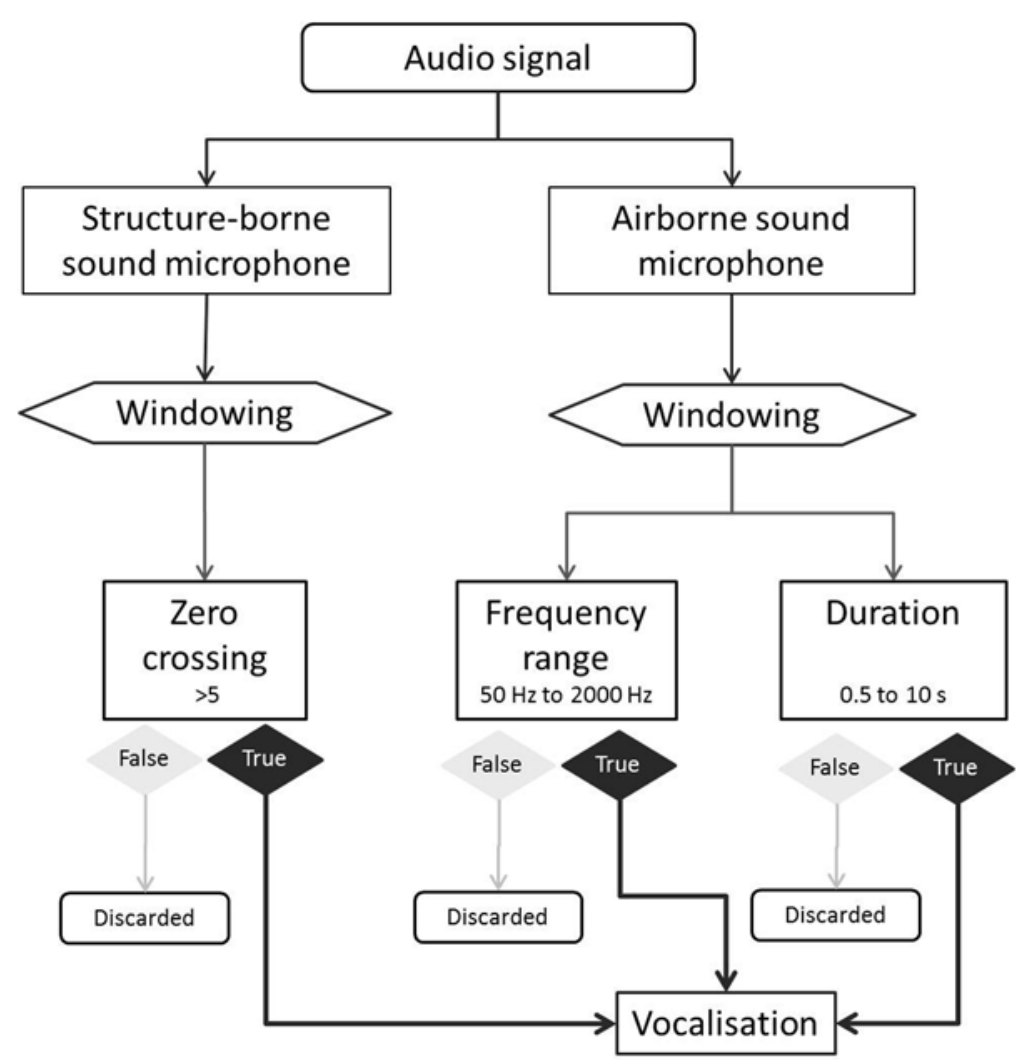

Figure 2 Process of call recording. Program flow chart of the developed algorithm for caller identification in dairy cattle (Bos taurus).

(a)

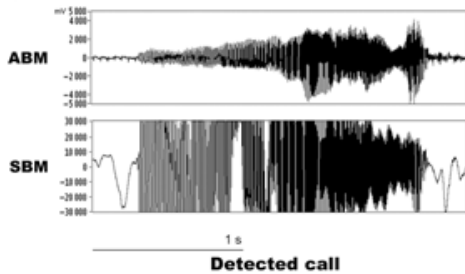

(b)

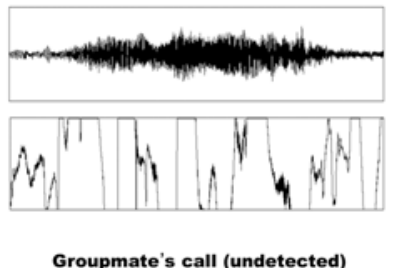

(c)

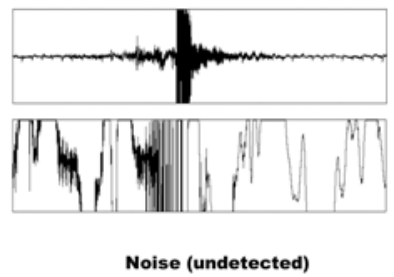

Figure 3 Visualisation of caller identification. The picture shows the time signals of the airborne sound microphone (ABM, top row) and the structure-borne sound microphone (SBM, bottom row) for three possible scenarios in dairy cattle (Bos taurus): (a) a correctly detected call of the focus animal (match of ABM and SBM), (b) a correctly undetected call emitted by a group mate (mismatch of ABM and SBM) and (c) noise (correctly undetected; mismatch of ABM and SBM).

Video recording was performed for $24 \mathrm{~h}$ on all analysed days. To synchronise the CCM data with the video data, a vocal timestamp was included on the audio stream shortly before the collars were reattached to the heifers (i.e., the experimenter announced the date, hour, minute and second, as given in the video live stream, and the subject's identification number).

Video analysis was performed with The Observer XT 10.1 software (Noldus Information Technology, Wageningen, the Netherlands) over a period of 5 days in the perioestrus and oestrus periods. Every vocalisation was coded as a point event, and the caller was identified (as the focus animal $v$. the group mates) based on characteristic head movements and/or exhalation of the focus animal that was synchronous with the vocalisation (Röttgen et al., 2018). These video- observed vocalisations were used as a gold standard for comparison to the machine-detected vocalisations.

Comparing video observation to machine-aided detection. The machine-detected and the video-observed vocalisations were first compared based on the timestamps in order to find the matching pairs. To do this, the CCM data were imported into the video observation files as external data.

Events recorded by the CCM and in the videos were classified into the following categories: (1) true positive: vocalisation of the focus animal was identified in the video and correctly detected by the CCM; (2) true negative: vocalisation by a group mate was identified in the video and was (correctly) not assigned to the focus animal by the CCM; (3) false positive: no vocalisation of the focus animal was identified in 
Röttgen, Schön, Becker, Tuchscherer, Wrenzycki, Düpjan and Puppe

the video, but vocalisation was incorrectly detected by the CCM and (4) false negative: vocalisation of the focus animal was identified in the video but was not identified by the CCM.

\section{Statistical analysis}

Vocalisations were excluded when they occurred during collar changes $(n=37)$ or one CCM malfunction $(n=53)$, where the microSD card was compromised and the data could not be accessed.

The following indices were calculated:

$$
\begin{gathered}
\text { sensitivity }=\frac{\text { true positive }}{\text { true positive }+ \text { false negative }} \times 100 \\
\text { specificity }=\frac{\text { true negative }}{\text { true negative }+ \text { false positive }} \times 100 \\
\text { positive predictive value }=\frac{\text { true positive }}{\text { true positive }+ \text { false positive }} \times 100
\end{gathered}
$$

negative predictive value $=\frac{\text { true negative }}{\text { true negative }+ \text { false negative }} \times 100$

$$
\text { detection level }=\frac{\text { true positive }}{\text { all vocalizations }} \times 100
$$

For split calls (see the 'Machine-aided vocalisation detection' section) the first 'call' was counted as a true positive, and the second 'call' was considered a false positive.

\section{Results}

In total, 2171 vocalisations were detected from the five focus animals and their group mates. Thereof, 1404 vocalisations were assigned to the focus animals by video observation and 721 vocalisations were emitted by respective group mates (Table 1).

The CCM operated continually for $24 \mathrm{~h}$ until the power supply changes and operated over the whole period of the pilot study. The system recorded $576 \mathrm{~h}$ of audio data, which were analysed by the algorithm. Only $24 \mathrm{~h}$ had to be omitted from the analyses due to the CCM malfunction. Technical problems that occurred during the testing of our prototype were the limited buffer capacity of the processing unit and the limited writing speeds of the microSD cards. These problems caused an offset between the timestamp of the audio file and the video timestamp. Additionally, these technical issues caused data gaps within and between files. The gaps between the files caused approximately $1 \%$ of the undetected vocalisations for the CCM.

During the observation period, 1404 vocalisations were observed from five heifers by analysing $576 \mathrm{~h}$ of audiovisual recordings, and 1266 vocalisations were detected by the algorithm in the same period (Table 1). Out of the 1404 vocalisations, $1220(87 \%)$ were detected by the CCM with correct
Table 1 Total number of vocalisations. Overview of the number of calls detected in dairy cattle (Bos taurus) by video observation and cattle call monitor (CCM) across subjects and the derived indices

\begin{tabular}{lccc}
\hline \hline & \multicolumn{2}{c}{ Video } & \\
\cline { 2 - 3 } Total: 2171 & Detected & Undetected & Predictive value \\
\hline CCM & & & \\
Detected & 1220 & 46 & $\begin{array}{c}\text { Positive } \\
96 \%\end{array}$ \\
Undetected & 184 & 721 & Negative \\
& & & $80 \%$ \\
& Sensitivity & Specificity & \\
& $87 \%$ & $94 \%$ & \\
\hline \hline
\end{tabular}

caller identification (true positives). On the other hand, 184 vocalisations were not detected by the CCM (false negatives). Among these, 82 vocalisations were clearly visible and audible in the video but could still not be found in the automatic detection data; another 81 vocalisations were 'moo'-type vocalisations that were (too) short and/or (too) quiet, and 6 vocalisations were throaty. In 15 cases, the vocalisations occurred in the gap between two data blocks.

The CCM detected 46 vocalisations that were not emitted by the focus heifer (false positives). Out of these vocalisations, 19 were vocalisations by group mates, 5 were probably vocalisations by group mates but occurred simultaneously with farm equipment noise, and the remaining 22 were split calls (see above).

These findings resulted in a sensitivity of $87 \%$, a specificity of $94 \%$, a negative predictive value of $80 \%$ and a positive predictive value of $96 \%$.

The detection rates varied between individual heifers, ranging from $60 \%$ to $100 \%$ (Table 2 ).

\section{Discussion}

In this study, a collar-based system for individual vocalisation detection in cattle was developed and validated. The results of the pilot study show that vocalisations during perioestrus and oestrus of individual animals can be detected with a sensitivity of $87 \%$ and a positive predictive value of $96 \%$. The specificity was $94 \%$, and the negative predictive value was $80 \%$.

Most of the undetected vocalisations were short and quiet mooing that most likely did not meet criteria 2 (duration of the ABM signal between 0.5 and $10 \mathrm{~s}$ ) and 3 (five zero crossings per window for the SBM signal). These sounds are produced with a closed mouth and minimal change in posture. While these vocalisations might be of interest in the context of animal welfare, in the context of oestrus detection, these vocalisations might be irrelevant. A study by Schön et al. (2007) showed a tendency towards more nonharmonic vocalisations on the day of oestrus compared to those on di-oestrus days. These nonharmonic calls differ in their structure 
Automatic vocalisation recording in cattle

Table 2. Individual detection rates and main detection errors. Individual call numbers, detection rates and the main detection errors (percentages unless indicated otherwise) of the five focus heifers (Bos taurus)

\begin{tabular}{|c|c|c|c|c|c|c|}
\hline & Detection errors & Animal A & Animal B & Animal C & Animal D & Animal \\
\hline Total number of detected calls (CCM + video) & & 1135 & 134 & 30 & 54 & 96 \\
\hline Number of CCM detected calls confirmed by video observation & & 1005 & 85 & 18 & 54 & 58 \\
\hline Detection rate & & 89 & 63 & 60 & 100 & 60 \\
\hline \multirow[t]{2}{*}{ CCM detected calls not confirmed by video observation } & & 1.5 & 2.2 & 40 & 0 & 13.5 \\
\hline & Split calls & 1.3 & 0 & 0 & 0 & 5.2 \\
\hline \multirow[t]{3}{*}{ Calls detected by video observation only } & & 10 & 34.3 & 0 & 0 & 26.0 \\
\hline & Quiet short moos & 3.1 & 28.4 & 0 & 0 & 8.3 \\
\hline & In a gap & 0.9 & 0 & 0 & 0 & 5.2 \\
\hline
\end{tabular}

CCM=cattle call monitor.

and length from the short and quiet mooing calls. The developed algorithm appears to have had problems detecting the mooing vocalisations. This is also apparent in the detection rates for the individual animals, for example, an animal that produced more mooing vocalisations than the others had the lowest detection rate of $60 \%$. The length of the calls being detected must be limited to suppress the detection of ambient sounds. During the development of the prototype, metallic sounds close to the SBM (e.g., caused by the self-locking feeding fence) were found to have great impact on that microphone, with amplitudes above the threshold $(1 \%$ of the maximal voltage of the $A B M$ ). The duration restriction is likely to have lowered the negative predictive value for these metallic sounds, as duration is the only criterion that discriminates these sound events from calls. This index gives the probability that a 'no vocalisation' readout from the CCM is correct. In contrast, the positive predictive value, which gives the probability that a registered vocalisation was indeed produced by the focus animal, was high. Therefore, since eliminating noise is crucial and the proportion of undetected mooing calls may not have an impact on oestrus detection (as nonharmonic calls seem to be more important to detect oestrus in cows), we see no need to alter the algorithm.

The second large group of undetected vocalisations included vocalisations that were clearly visible/audible and assignable to the focus animal. The reason that these vocalisations were not detected by the CCM might be that the SBM was not attached properly to the body of the heifer or that a data gap occurred while the data were written to the memory card during the time of the vocalisation. The contact between the heifer's neck and the SBM was sufficient in most cases, but contact can be impaired during the contraction of specific neck muscles. This problem can only be solved if the SBM is permanently and directly mounted on the skin or with a much tighter-fitting collar. A permanent solution can only be achieved by implanting or stitching the microphone to the animal. However, this solution would not be feasible for on-farm use. A tighter fitting collar would probably result in mechanical problems during feed intake due to the constriction of the oesophagus. Additionally, a tighter collar might cause venous stasis of the jugular vein and mechanical skin erosions. Hence, given the high overall sensitivity and the significant side effects of possible solutions that could impair animal welfare, we consider this inaccuracy negligible.

The results showed two kinds of data gaps: the first kind was within a file and the second kind was between files. Gaps within files were difficult to reconstruct with an accurate timeline, so vocalisations that were missing in the automatic detection data as a result of such gaps were counted as mismatches. The gaps between files were, however, easy to reconstruct, and the vocalisations that were emitted during these gaps were excluded. To solve the problem of data gaps, technical improvements must be made. One approach could be to integrate the algorithm into the collar-mounted processing unit itself and to relay the audio data stream without previous saving. This would also provide considerable progress towards developing an applicable system for on-farm use.

Some vocalisations were divided into two parts because one window failed to match the recording criteria, but a subsequent window during that vocalisation fulfilled the criteria. This caused a split recording of these vocalisations. One potential adjustment to the algorithm could be to make it end the recording only if two or more consecutive windows fail the criteria.

Once these technical problems are solved, analysis of algorithms for oestrus detection must begin. These algorithms need to identify peaks in individual vocalisation rates based on the CCM data and must therefore be robust against significant individual variations in vocal behaviour. Our focus animals showed considerable variation in individual vocalisation rates, but our data do not indicate that these influence the reliability of call detection (with the highest detection rate of $100 \%$ in an animal with 54 vocalisations and the second highest detection rate of $89 \%$ in an animal with 1135 total detected calls). An adaptive phase is the approach used to deal with such individual vocalisation rates. In this phase, the data from the CCM would be analysed and the mean vocalisation rate per time interval calculated for each individual cow. Significant increases in mean vocalisation rate could then indicate oestrus for that particular animal. A higher number of vocalisations are also observed in stressful situations (Stookey et al., 1996; Grandin, 2001; Green et al., 2018). These events should also be considered in the 
algorithm, for example, by comparing the data to the date of the last oestrus event (adequate time span) and/or by crosschecking the data with that of herd members during raw data processing. This would exclude the increased number of vocalisations that can occur during routine events such as regrouping or routine treatments (e.g., hoof trimming) that affect the whole herd.

Various technical approaches have been used to assess the vocalisations of animals, particularly those of farm animals. Yajuvendra et al. (2013) chose to detect individual cows by applying speech analysis techniques to samples of cattle vocalisations. This approach requires a set of sample vocalisations for each animal, so that the selected parameters can be recorded. During routine daily farm work, such samples are hard to obtain. Aside from the fact that cattle rarely vocalise, there are also many ambient sounds on a farm throughout the day, such as those emitted by other animals, farm equipment and personnel. It would also be timeconsuming to obtain vocalisation samples for each cow and would therefore not be economically viable. In chipmunks, Couchoux et al. (2015) attached microphones to the necks of the animals and were able to record their vocal behaviours throughout the day. This approach does not require a set of individual vocalisation samples, and it can record all vocalisations of the focus animal. However, the problem with using a single microphone in a farm environment is that many animals of the same species live in a limited space, and other than in zebra finches (Gill et al., 2016), the use of amplitude differences for caller identification is not applicable in dairy cattle. Therefore, techniques used in free-ranging solitary-living animals or songbirds would need to be adapted for on-farm application. The CCM relies neither on individual vocalisation samples nor on isolated individual housing, increasing its applicability on-farm.

A technical acoustic solution to monitor coughing in pigs involves installing multiple microphones, which enables detection of the compartment where the cough was emitted by triangulation (Silva et al., 2008). Meen et al. (2015) suggested a similar system for cows to monitor their vocalisations. However, in the context of oestrus detection, the localisation of the housing compartment is not sufficient, as the individual cow must be identified, for example, for artificial insemination. Another problem with this approach is the lack of a specific call indicating oestrus in cows. It has been proven only that the vocalisation rate rises on the day of oestrus (Schön et al., 2007; Dreschel et al., 2014; Röttgen et al., 2018); individual vocalisation rates show a significant variation both within and between animals. Even if further studies identify a specific vocalisation or a specific change in vocalisation patterns during oestrus, individual assignment would remain a challenge.

The CCM could also be integrated into or combined with existing oestrus detection devices that measure other behaviours, for example, physical activity or rumination. Synchronously monitoring more than one behavioural parameter would likely result in a higher accuracy of oestrus detection in cattle (Reith and Hoy, 2018) and might be especially effective in cases where current methods fail (e.g., 'silent heat'). Additionally, analysis of the sequence of behaviours occurring during oestrus in cattle might enable improvements. For example, the climax of the vocalisation rate is almost synchronous with the climax of oestrus behaviour (Röttgen et al., 2018), and both are synchronous with the oestradiol peak (Lyimo et al., 2000), whereas the climax in physical activity appears 6 to $12 \mathrm{~h}$ after the oestradiol peak (Aungier et al., 2015). Monitoring vocalisation could therefore provide farmers with early information that can be substantiated by later observations of increased physical activity.

\section{Conclusion}

As a whole, the CCM has the potential to record cattle vocalisations and to correctly identify the calling individual in commercial housing environments. The automatic assignment of vocalisations to an individual animal and a specific time point will limit the time required for video analysis searching for rare cattle vocalisations and will therefore enhance progress towards decoding specific vocalisations. Such scientific findings could be implemented in the CCM to further improve its on-farm applicability. These results are promising for the future use of vocalisation in automatic oestrus detection systems and for further applications in monitoring animal health and welfare status.

\section{Supplementary material}

To view supplementary material for this article, please visit https://doi.org/10.1017/S1751731119001733

\section{Acknowledgements}

The authors thank the staff of the experimental facility for cattle of the Leibniz Institute for Farm Animal Biology and the technicians of the Institute of Behavioural Physiology and Institute of Reproductive Biology for their admirable penitence and support during the development. The funding of this project by the European Social Fund MecklenburgWestern Pomerania (V-630-F-124-2011/150 V-630-S-1242011/151) was greatly appreciated. Two anonymous reviewers helped increasing the clarity of the manuscript with their valuable input.

\section{Declaration of interest}

The authors have no conflicting interests to disclose.

\section{Ethics statement}

The experiments were approved by the federal state of Mecklenburg-Western Pomerania (LALLF M-V/TSD/7221.3-2.1021/13) according to German regulations.

\section{Software and data repository resources}

All raw data can be provided on request. 


\section{References}

Aungier SPM, Roche JF, Duffy P, Scully S and Crowe MA 2015. The relationship between activity clusters detected by an automatic activity monitor and endocrine changes during the periestrous period in lactating dairy cows. Journal of Dairy Science 98, 1666-1684. https://doi.org/10.3168/jds.20137405.

Couchoux C, Aubert M, Garant D and Reale D 2015. Spying on small wildlife sounds using affordable collar-mounted miniature microphones: an innovative method to record individual daylong vocalisations in chipmunks. Scientific Reports 5, 10118. https://doi.org/10.1038/srep10118.

Dreschel S, Schön PC, Kanitz W and Mohr E 2014. Vocalization of dairy cattle during the oestrous cycle in two different housing systems. Züchtungskunde 86, 157-169.

Düpjan S, Schön PC, Puppe B, Tuchscherer A and Manteuffel G 2008. Differential vocal responses to physical and mental stressors in domestic pigs (Sus scrofa). Applied Animal Behaviour Science 114, 105-115. https://doi.org/10.1016/j. applanim.2007.12.005\#.

Fischer J, Kitchen DM, Seyfarth RM and Cheney DL 2004. Baboon loud calls advertise male quality: acoustic features and their relation to rank, age, and exhaustion. Behavioral Ecology and Sociobiology 56, 140-148. https://doi. org/10.1007/s00265-003-0739-4.

Gill LF, D'Amelio PB, Adreani NM, Sagunsky H, Gahr MC and Maat A 2016. A minimum-impact, flexible tool to study vocal communication of small animals with precise individual-level resolution. Methods in Ecology and Evolution 7, 1349-1358. https://doi.org/10.1111/2041-210X.12610.

Grandin T 2001. Cattle vocalizations are associated with handling and equipment problems at beef slaughter plants. Applied Animal Behaviour Science 71, 191-201. https://doi.org/10.1016/S0168-1591(00)00179-9.

Green AC, Johnston IN and Clark CEF 2018. Invited review: the evolution of cattle bioacoustics and application for advanced dairy systems. Animal 12, 1250-1259. https://doi.org/10.1017/S1751731117002646.

Leliveld LMC, Düpjan S, Tuchscherer A and Puppe B 2016. Behavioural and physiological measures indicate subtle variations in the emotional valence of young pigs. Physiology \& Behavior 157, 116-124. https://doi.org/10.1016/j. physbeh.2016.02.002.

Leliveld LMC, Düpjan S, Tuchscherer A and Puppe B 2017. Vocal correlates of emotional reactivity within and across contexts in domestic pigs (Sus scrofa). Physiology \& Behavior 181, 117-126. https://doi.org/10.1016/j.physbeh.2017. 09.010 .

Lyimo ZC, Nielen M, Ouweltjes W, Kruip TAM and van Eerdenburg FJCM 2000 Relationship among estradiol, cortisol and intensity of estrous behavior in dairy cattle. Theriogenology 53, 1783-1795. https://doi.org/10.1016/S0093-691X(00) 00314-9.

Meen GH, Schellekens MA, Slegers MHM, Leenders NLG, van Erp-van der Kooij E and Noldus LPJJ 2015. Sound analysis in dairy cattle vocalisation as a potential welfare monitor. Computers and Electronics in Agriculture 118, 111-115. https://doi.org/10.1016/j.compag.2015.08.028.
Mielke A and Zuberbühler K 2013. A method for automated individual, species and call type recognition in free-ranging animals. Animal Behaviour 86 475-482. https://doi.org/10.1016/j.anbehav.2013.04.017.

Pitcher BJ, Briefer EF, Vannoni E and McElligott AG 2014. Fallow bucks attend to vocal cues of motivation and fatigue. Behavioral Ecology 25, 392-401. https:// doi.org/10.1093/beheco/art131.

Puppe B, Schön PC, Tuchscherer A and Manteuffel G 2005. Castration-induced vocalisation in domestic piglets, Sus scrofa: complex and specific alterations of the vocal quality. Applied Animal Behaviour Science 95, 67-78. https://doi.org/ 10.1016/j.applanim.2005.05.001.

Reith S, Fengels I and Hoy S 2012. Investigations on heat detection in dairy cows by automatically measured rumination. Züchtungskunde 84, 281-292.

Reith S and Hoy S 2018. Review: behavioral signs of estrus and the potential of fully automated systems for detection of estrus in dairy cattle. Animal 12 398-407. https://doi.org/10.1017/S1751731117001975.

Röttgen V, Becker F, Tuchscherer A, Wrenzycki C, Düpjan S, Schön PC and Puppe B 2018. Vocalization as an indicator of estrus climax in Holstein heifers during natural estrus and superovulation. Journal of Dairy Science 101, 2383-2394. https://doi.org/10.3168/jds.2017-13412.

Rutten CJ, Velthuis AGJ, Steeneveld W and Hogeveen H 2013. Invited review: sensors to support health management on dairy farms. Journal of Dairy Science 96, 1928-1952. https://doi.org/10.3168/jds.2012-6107.

Schön PC, Hämel K, Puppe B, Tuchscherer A, Kanitz W and Manteuffel G 2007. Altered vocalization rate during the estrous cycle in dairy cattle. Journal of Dairy Science 90, 202-206. https://doi.org/10.3168/jds.S0022-0302(07)72621-8.

Schön PC, Puppe B and Manteuffel G 2001. Linear prediction coding analysis and self-organizing feature map as tools to classify stress calls of domestic pigs (Sus scrofa). Journal of the Acoustical Society of America 110, 1425-1431. https:// doi.org/10.1121/1.1388003

Schön PC, Puppe B and Manteuffel G 2004. Automated recording of stress vocalisations as a tool to document impaired welfare in pigs. Animal Welfare 13, 105-110.

Silva M, Ferrari S, Costa A, Aerts JM, Guarino M and Berckmans D 2008. Cough localization for the detection of respiratory diseases in pig houses. Computers and Electronics in Agriculture 64, 286-292. https://doi.org/10.1016/j.compag. 2008.05.024.

Stookey J, Watts J and Schwartzkopf K 1996. Effects of restraint and branding on subsequent ease of movement through a chute in beef cattle. Journal of Animal Science 74 (suppl. 1), 133.

Walker WL, Nebel RL and McGilliard ML 1996. Time of ovulation relative to mounting activity in dairy cattle. Journal of Dairy Science 79, 1555-1561. https://doi.org/10.3168/jds.S0022-0302(96)76517-7.

Yajuvendra S, Lathwal SS, Rajput N, Raja TV, Gupta AK, Mohanty TK, Ruhil AP, Chakravarty AK, Sharma PC, Sharma V and Chandra G 2013. Effective and accurate discrimination of individual dairy cattle through acoustic sensing. Applied Animal Behaviour Science 146, 11-18. https://doi.org/10.1016/j.applanim.2013. 03.008 . 